\title{
Connectives and narrative text: The role of continuity
}

\author{
JOHN D. MURRAY \\ Georgia Southern University, Statesboro, Georgia
}

\begin{abstract}
Connectives are text devices that signal the relation between adjacent sentences. Recently there has been a surge of research interest in the role played by connectives in on-line processing. The present research tested the hypothesis that connectives will impact on-line processing to the extent that they signal a text event that represents a departure from the continuity of the events stated in the text. In Experiment 1, participants generated sentences to follow a stimulus sentence. An additive, causal, or adversative connective (or no connective) was provided to serve as the first word of the participants' sentence. Results showed that sentences generated in response to additive or causal connectives depicted text events that were continuous with the stimulus text. In contrast, sentences generated in response to adversative connectives depicted discontinuous text events. In Experiments 2 and 3, participants read coherent sentence pairs containing inappropriately placed additive, causal, or adversative connectives. Support for the continuity hypothesis was found when it was shown that adversative connectives led to the greatest amount of processing disruption, as measured by longer reading time on the postconnective sentence (Experiment 2) and lower ratings of coherence (Experiment 3 ). Future research in this area is discussed.
\end{abstract}

Connectives are text devices that explicitly mark the relation between adjacent text units. Terms like because, and, but, thus, also, and however are examples of connectives that are often placed between independent clauses. Connectives are ubiquitous in both narrative and expository text, and several researchers have examined how connectives affect on-line processing and comprehension (Caron, Micko, \& Thuring, 1988; Golding, Millis, Hauselt, \& Sego, 1994; Goldman \& Murray, 1992; Haberlandt, 1982; Kinstch \& van Dijk, 1978; Millis, Graesser, \& Haberlandt, 1993; Millis \& Just, 1994). The growing number of research investigations devoted to studying connectives indicates the importance that researchers place on them in reading. Researchers in language development, too, have shown interest in this topic, investigating, for example, how children and non-native speakers of English acquire connectives (Bloom, Lahey, Hood, Lifter, \& Fiess, 1980; Katz \& Brent, 1968; Lintermann-Rygh, 1985; McClure \& Geva, 1983; Peterson \& McCabe, 1987).

Ironically, the empirical attention devoted to the processing and acquisition of connectives stands in stark contrast to the lack of attention to connectives from reading researchers. With respect to this issue, Segal, Duchan, and Scott (1991) reviewed several reading models and

Experiment 2 was presented at the 1993 annual meeting of the Psychonomic Society in Washington, DC. Thanks go to A. Deal, B. MacIntyre, H. Jackson, K. Hobbs, and B. Kaye for help with data scoring Also, thanks go to E. Saul, J. Myers, C. Klin, J. Steirn, and two anonymous reviewers for comments on earlier versions of this manuscript. Correspondence should be addressed to J. D. Murray, P.O. Box 8041, Department of Psychology, Georgia Southern University, Statesboro, GA 30460-8041 (e-mail: jmurray@gasou.edu). showed that virtually none make reference to how readers make use of connectives to facilitate the construction of a cognitive representation of the text. This lack of attention is clearly in error since connectives provide an obviously critical function of informing readers how adjacent text units are related (Halliday \& Hasan, 1976). Furthermore, in a recent paper, Millis and Just (1994) argued that connectives play a dynamic role in on-line comprehension processes by "modulating the activation of constituent structures needed to arrive at a coherent model of the discourse" (p. 145).

The increased attention to connectives has led to evidence in support of their processing benefits. Experiments have shown that the presence of a connective between adjacent clauses reduces the reading time of the second clause (Haberlandt, 1982; Millis \& Just, 1994), enhances memory for the clauses (Caron et al., 1988; but see Millis et al., 1993), increases accuracy in response to comprehension questions, and decreases question-answering time (Millis \& Just, 1994). Furthermore, the rules underlying the usage of different connectives are accessible and relatively easy to articulate (Goldman \& Murray, 1992), the implication being that knowledge of connectives and of constraints in their usage may be helpful during reading.

Despite the view that connectives facilitate comprehension, what remains unclear is how different types of connectives impact processing. Research has presented an equivocal picture on this issue. For example, Caron et al. (1988) showed that memory for sentence pairs was improved when because was placed between the sentences but not when and or but was placed between the sentences, suggesting that causal connectives result in better sen- 
tence integration than do additive or adversative connectives. In contrast, Murray (1994) showed that adversative connectives were associated with better memory than were additive and causal connectives. When reading time of the line following a connective was measured, both Haberlandt (1982) and Millis and Just (1994) found that shorter reading times were associated with the presence of both causal and adversative connectives. Murray's research, on the other hand, showed that only adversative connectives significantly decreased reading time.

This equivocal picture is undoubtedly due, in part, to differences across the experiments in the types of experimental stimuli and procedures used. However, the fact that differences continue to emerge among different types of connectives in terms of their on-line benefits indicates that not all connectives affect sentence processing and integration in the same manner. If reading researchers wish to understand how connectives affect processing, research is needed that focuses on how specific types of connectives facilitate text comprehension. The present research was conducted with this goal in mind.

The present research was conducted in a highly common text setting, one in which the sentences surrounding the connective convey coherent semantic relations and are easy to comprehend. Specifically, three types of interclause relations were studied: Additive relations are those where the second sentence elaborates in a nonspecific manner on the content of the first sentence. Causal relations indicate simple cause-effect relations between the sentences. Adversative relations indicate that the second sentence contrasts or limits the scope of the content of the first sentence.

Recently, Murray (1994) used these types of materials to investigate how additive (e.g., and), causal (e.g., so), and adversative (e.g., but) connectives affect the on-line processing of sentence pairs. Participants read sentence pairs where a semantically appropriate connective was placed in the initial position of the second sentence or was absent. The reading time (RT) of the second sentence and memory for the sentence pair were measured. A synopsis of these results can be seen in Table 1. In two experiments, the presence of an adversative connective led to shorter reading time on the second sentence and better memory for the sentence pair than when the connective was absent. ${ }^{1}$ However, such a benefit was not observed with additive or causal connectives. Murray interpreted these findings as indicating that connectives differ in the extent to which they impact interclause cohesion, at least when they appear between cohesive sentences (see also Segal, Duchan, \& Scott, 1991). Specifically, adversatives play a more critical role in maintaining and facilitating interclause cohesion than do additives or causals.

The purpose of the present research was to explore the psychological mechanism underlying the differential contributions made by additive, causal, and adversative connectives to integrative processes. The proposed mechanism is heavily influenced by two related perspectives on reading: (1) that narrative text processing consists of an attempt by the reader to construct a mental model of the world conveyed by the text (Johnson-Laird, 1983), and (2) that readers keep track of the here-and-now from some perspective, such as from a character or from the narrator. This latter perspective is known as the "deictic shift" perspective and has been described in detail in Bruder et al. (cited in Segal et al., 1991). According to mental model theory, readers' memory representation of a narrative is composed of an interpretation of the text events being depicted rather than a strictly linguistically based representation. The deictic shift perspective expands on mental model theory by postulating that the reader processes a narrative from the point of view within the story (i.e., from the perspective of the narrator, protagonist, or another character within the story). Consequently, the reader processes the narrative within the world of the story, rather than as an external viewer.

The relevance of this perspective to the present research is the principle of continuity, which lies at the heart of deictic shift theory. This principle states that readers have a bias toward interpreting sentences in a narrative as following one another in a continuous manner. As readers progress through a narrative, they assume that the events will follow in a linear fashion. And when this occurs, reading is relatively easy. Continuity can be conveyed easily via additive or causal relations. When a reader encodes a text event that is discontinuous in the absence of a marker or indication of the discontinuity, reading is more difficult. Examples of discontinuity are numerous and include reversions to an earlier setting or scene (such as a flashback), an abrupt topic change, a surprising turn of events, a character moving away from what he/she is currently doing, or a violation of an expectation created in the previous text. In their research, Segal et al. (1991) had participants classify the nature of the relation between successive sentences in a discourse and found that the majority of sentences reflected a continuous relationship from the previous sentence. They interpreted this finding

Table 1

Mean Reading Times (RT; in Milliseconds) of Postconnective Sentence and Mean Number of Items Recalled (Memory) Out of 10 Items

\begin{tabular}{|c|c|c|c|c|c|c|c|c|}
\hline \multirow[b]{3}{*}{ Group } & \multicolumn{8}{|c|}{ Type of Relation } \\
\hline & \multicolumn{2}{|c|}{ Additive } & \multicolumn{2}{|c|}{ Causal } & \multicolumn{2}{|c|}{ Adversative } & \multicolumn{2}{|r|}{$M$} \\
\hline & RT & Memory & RT & Memory & RT & Memory & $\mathrm{RT}$ & Memory \\
\hline Connectives present & 2,132 & 4.3 & 2,063 & 6.6 & 2,080 & 7.3 & 2,092 & 6.1 \\
\hline Connectives absent & 1,980 & 5.4 & 1,960 & 6.2 & 2,253 & 7.3 & 2,064 & 6.3 \\
\hline
\end{tabular}

Note-From "Logical Connectives and Local Coherence," by J. D. Murray, in R. F. Lorch and E. J. O'Brien (Eds.) (1994), Sources of Cohesion in Text Comprehension (pp. 112-113), Hillsdale, NJ: Erlbaum. Copyright 1994 by Lawrence Erlbaum. Adapted with permission. 
as indicative of readers' bias to perceive continuity in narrative discourse.

In the present research, a mechanism was investigated that may account for the different effects of additive, causal, and adversative connectives and that also pertains directly to the principle of continuity. First, it is proposed that logical connectives serve as explicit markers of continuity and discontinuity in a discourse. Second, and more importantly, it is proposed that connectives differentially facilitate on-line processing to the extent they inform the reader about an upcoming discontinuity. If readers assume by default that text events are continuous, connectives that signal continuity should have less of an impact on on-line processing than those that signal a break in continuity. Specifically, connectives that signal a break in continuity would be predicted to facilitate integrative processing (i.e., cause shorter RT on the subsequent line; lead to better integration of the clauses surrounding the connective) to a greater extent than connectives that signal continuity.

This proposal, termed the "continuity hypothesis," predicts that additive and causal connectives should lead to less processing facilitation than adversative connectives because the former indicate continuity in the discourse whereas the adversatives indicate discontinuity. This hypothesis is consistent with Murray's (1994) findings.

It is unclear whether the continuity hypothesis can explain the findings of other researchers because it has not been adequately tested. For example, in their research, Caron et al. (1988) used pairs of sentences that did not convey a sensible relation to each other. Perhaps in order to make sense of the experimental task, participants employed special strategies to forge a connection between the sentences. Furthermore, the finding that $b e-$ cause facilitates recall of sentence pairs better than and or but is not inconsistent with the continuity hypothesis. Whereas because indicates a causal relation between two text events, this relation departs from individuals' normative perceptual experiences of events following a cause-effect sequence. For example, in the sentence, "Mark gave his dog a bath because the dog had fleas," the clause that follows because is the cause of the clause preceding the connective. In contrast, a connective like therefore, so, or thus indicates a cause-effect flow of events, a pattern that maps more closely onto personal experience. The continuity hypothesis would predict that a connective like because would be more facilitatory than a connective like therefore because it signals a nonlinear flow of events.

Millis and Just (1994) found shorter RTs on the second sentence after the adversative connective although as well as after because. The facilitation observed with although is consistent with the continuity hypothesis, and for the reason cited above, because should also lead to reading facilitation.

Finally, Haberlandt (1982) found that causal and adversative connectives decreased reading time on the subsequent line to an equal extent. However, Haberlandt's experiment involved measuring RT on a sentence that followed the aggregate content of the previous four sentences. In its present form, the continuity hypothesis predicts when connectives will facilitate the integration of pairs of sentences. It may be the case that the impact of connectives changes when readers are integrating a single sentence with a larger text unit.

In a further effort to explore the continuity hypothesis, Experiment 1 tested whether connectives differ in the extent to which they signal continuous and discontinuous text events. Participants provided one-sentence continuations in response to single sentences followed by an additive, causal, or adversative connective, or no connective. In accordance with the continuity hypothesis, additive connectives and causals that signal cause-effect relations were expected to elicit text events that were continuous with the event depicted in the stimulus sentence. In contrast, adversatives were expected to elicit text events that were discontinuous with the stimulus sentence.

Experiments 2 and 3 employed the materials used in earlier research (Murray, 1994) to test the implications of the continuity hypothesis for on-line processing. Murray showed that, when used appropriately, connectives signaling discontinuity led to greater processing facilitation than connectives signaling continuity. Experiments 2 and 3 put the continuity hypothesis to a more specific test: If signals of discontinuity are the more informative, then their inappropriate placement should lead to greater processing disruption than inappropriately placed signals of continuity. To this end, participants read coherent sentence pairs containing inappropriately placed connectives. In Experiment 2, participants were timed in reading the clause following the connective, and in Experiment 3 participants rated the degree to which the two clauses were coherent. One reason for using a coherence rating task in Experiment 3 was to rule out the possibility that participants' reading times in Experiment 2 were heavily influenced by factors other than the inappropriate connective. In both Experiments 2 and 3, it was expected that all connectives would produce interference (i.e., longer RTs and lower coherence ratings compared to the no-connective absent condition). Interference was expected from all inappropriately placed connectives because the second sentence violates the continuity or discontinuity signaled by the connective. However, the primary prediction was that adversative connectives would produce significantly more interference than the additive or causal connectives.

\section{EXPERIMENT 1}

\section{Method}

Participants. Forty-four undergraduates at Georgia Southern University participated in the experiment in exchange for extra credit in an introductory psychology course.

Materials. Twenty-eight unrelated sentences were taken from the materials used in Murray's (1994) research. Each sentence conformed to a simple subject-predicate format, as in the sentence "Ronny cleaned up the house for his girlfriend's visit" Four versions of each sentence were created. Each version consisted of the 
sentence itself ending in a period followed by a blank line to be used by participants to write another sentence. The no-connective version was exactly as just described. The additive connective version was identical to the no-connective condition, except that an additive connective was printed at the beginning of the blank line. The first letter of the connective was capitalized, signifying that it was to be the first word in the sentence to be generated. The causal and adversative connective versions were similar except that a causal or adversative connective was placed at the beginning of the blank line. The additive connectives used in the present research were moreover, furthermore, also, and and. All causal connectives that were used indicated cause-effect relations and were therefore, so, thus, and consequently. The adversative connectives were yet, nevertheless, however, and but. The particular connectives selected from each category represented a comparable range of frequency of usage in English (Francis \& Kučera, 1982). One connective from each group was of relatively low frequency; two were at moderate levels; and one was at a relatively high level. Each connective was used an equal number of times over all 28 items.

Four booklets were constructed, and each booklet contained one of the versions of each item. Items were counterbalanced over condition, and each booklet contained seven items in each condition. Items were randomly ordered in the booklets.

Procedure. Participants were run in groups in a large classroom. Each participant was given a booklet containing the 28 items, and for each was instructed to generate a sentence that followed sensibly from the sentence provided. On the majority of the items, they were to use the word provided as the first word in their sentence. An unlimited amount of time was allowed for this task, but most participants completed the task in 30 min or less.

\section{Results and Discussion}

Participants' responses to each of the 28 items were transcribed directly from the booklets, and the connective was omitted in those items where it was provided. Each response was classified according to its relation to its stimulus sentence. Classification categories were derived from Halliday and Hasan (1976). A response was classified as "additive" if it elaborated on the stimulus sentence in a nonspecific way by stating additional related information. A response was classified as "causal" if it provided a result, effect, or logical conclusion from the stimulus sentence. A response was classified as "adversative" if it stated a comparison or contrast with the information in the stimulus sentence or reflected unexpected information. Any responses that did not fit into any of the categories above were classified as "miscellaneous." Items not responded to were coded as "no response."

Two independent coders, blind to the hypotheses of the experiment, classified the responses of 7 participants.
Agreement between the coders was $82.1 \%$. All disagreements were resolved by discussion. Next, the coders classified the responses of 4 additional participants. Agreement rose to $88.4 \%$. After the disagreements were resolved by discussion, one of the coders classified the remaining protocols.

The proportion of responses in each condition falling into each of the coding categories is displayed in Table 2. Only minimal differences emerged among the four conditions in the proportion of responses classified as miscellaneous and no response. A one-way analysis of variance (ANOVA) revealed no difference among the four conditions in the proportions classified as miscellaneous $[F(3,129)<1]$ or no response $[F(3,129)=2.03, p>.05]$; therefore, the proportions for these categories are combined in Table 2 .

The data in Table 2 indicate that participants were relatively sensitive to the meanings of the connectives, a finding consistent with prior research on connectives (e.g., Goldman \& Murray, 1992). The largest proportion of responses in each condition fell into the coding category indicating responses that were "appropriate to the connective." In the no-connective condition, the majority of the responses fell into the causal category followed by the additive category, a finding consistent with the continuity hypothesis. When left with no text cues indicating the relation of the upcoming text with the previous text, participants generated responses that were continuous with the content of the stimulus sentence.

In the three connective conditions, the proportions of responses classified in the condition-appropriate category were compared in an ANOVA. Unless otherwise noted, a significance level of .05 was adopted for the present analyses. A significant difference emerged among the conditions $\left[F(2,86)=20.53, M S_{\mathrm{e}}=.052\right]$. Pairwise contrasts (family-wise $\alpha=.017$ ) revealed that participants' responses were more often appropriate to adversative connectives $(.768)$ than to either additive $(.575)[F(1,43)=$ $\left.14.51, M S_{\mathrm{e}}=.113\right]$ or causal $(.459)$ connectives $[F(1,43)$ $\left.=45.88, M S_{\mathrm{e}}=.092\right]$. No difference was found between the proportions of responses appropriate to additive and causal conditions $(p=.024)$. This pattern indirectly supports the continuity hypothesis in that adversative connectives may be more salient than additive or causal connectives in the interclausal relations that they signal. Because discontinuity in a discourse is a rarer occurrence

Table 2

Mean Proportion and Standard Deviations of Responses Classified in Each Coding Category for Each Condition

\begin{tabular}{|c|c|c|c|c|c|c|c|c|}
\hline \multirow[b]{3}{*}{ Condition } & \multicolumn{8}{|c|}{ Coding Category } \\
\hline & \multicolumn{2}{|c|}{$\mathrm{ADD}$} & \multicolumn{2}{|c|}{ CAU } & \multicolumn{2}{|c|}{ ADV } & \multicolumn{2}{|c|}{$\mathrm{MISC}+\mathrm{NR}$} \\
\hline & $M$ & $S D$ & $M$ & $\overrightarrow{S D}$ & $M$ & $S \overline{S D}$ & $M$ & $S D$ \\
\hline Additive & .575 & .238 & .250 & .204 & .115 & .143 & .056 & .178 \\
\hline Causal & .330 & .231 & .459 & .248 & .158 & .147 & .042 & .173 \\
\hline Adversative & .130 & .130 & .082 & .117 & .768 & .234 & .030 & .153 \\
\hline No connective & .282 & .196 & .472 & .230 & .247 & .177 & .040 & .165 \\
\hline
\end{tabular}

Note-ADD, additive; CAU, causal; ADV, adversative; MISC, miscellaneous; NR, no response. 
than continuity (Segal et al., 1991), readers may notice and respond more accurately to connectives that signal discontinuity than to connectives that signal continuity.

Of primary interest was the extent to which the different connectives elicited continuous and discontinuous responses. For each of the three connective conditions, the proportion of responses classified as additive and causal were combined to create a single category that reflected the proportion of responses that were continuous with the stimulus sentence. For additive connectives, this proportion was .826; for causal connectives, it was .789; and for adversative connectives, it was .212. These proportions were significantly different $\left[F(2,86)=130.46, M S_{\mathrm{e}}=\right.$ .04]. Pairwise contrasts confirmed a priori expectations. Additive and causal connectives elicited a significantly higher proportion of responses reflecting continuity than did adversative connectives $[F \mathrm{~s}(1,43)=255.05$ and 133.8 , respectively, $M S_{\mathrm{e}} \mathrm{s}=.065$ and .11 , respectively]. Also as predicted, additive and causal connectives did not elicit reliably different proportions of continuous responses $[F(1,43)<1]$.

To examine the extent to which the different connectives elicited responses that were discontinuous with the stimulus sentence, the proportion of participants' responses classified as adversative were compared across the three connective conditions. Again, as expected, a significant difference emerged $\left[F(2,86)=217.03, M S_{\mathrm{e}}=\right.$ $.027]$. The results of pairwise contrasts showed that adversative connectives elicited a greater proportion of responses that were discontinuous with the prior text than either additive or causal connectives $[F \mathrm{~s}(1,43)=363.83$ and 228.24, respectively, $M S_{\mathrm{e}} \mathrm{s}=.05$ and .07 , respectively]. Furthermore, additive and causal connectives did not differ in the proportion of discontinuous responses that were elicited $\left[F(1,43)=2.02, M S_{\mathrm{e}}=.04\right]$.

The data from this experiment lend support to the notion that connectives are powerful indicators of continuity and discontinuity in text. Furthermore, connectives clearly differ in terms of whether they predominantly signal continuity or discontinuity. As previous research has shown, additive and causal connectives (i.e., causals that signal cause-effect relations) indicate that the next clause will continue the text event stated in the previous clause in a forward, linear manner. Adversatives, in contrast, indicate a disruption in continuity. That participants interpreted the lack of a connector as a cue of continuity supports the notion that readers' default assumption in reading is that subsequent sentences follow continuously from one another.

\section{EXPERIMENT 2}

In Experiment 2, participants read a series of sentence pairs that conveyed additive, causal, or adversative relations. Two thirds of the sentence pairs contained a logical connective between the sentences that was incongruent with the relation conveyed by the sentences. The remaining third contained no connective. The RT of the second sentence was measured. In addition, as was done in Mur- ray (1994), memory for the sentence pair was measured in a cued-recall task following the reading session. It was predicted that the presence of an inappropriate logical connective should lead to longer RT on the second sentence and poorer memory for the sentence pair compared with the no-connective condition. However, according to the continuity hypothesis, inappropriate adversative connectives should cause greater processing disruption than inappropriate additive or causal connectives.

\section{Method}

Participants. Forty-five undergraduates enrolled in introductory psychology participated in the experiment as a course requirement.

Materials. The primary stimuli were 30 sentence pairs used previously in Murray (1994). Each pair had three versions. Each version differed in the type of relation between the two sentences. See Table 3 for several sample items. Across the three versions, the first sentence differed and the second (the target) was identical. In the additive version, the target sentence elaborated in a nonspecific way on the first sentence; in the causal version, the target sentence stated an effect or consequence of something stated in the first sentence; in the adversative version, the target contrasted with the content of the first sentence. The first sentence in the three versions was comparable in length and wording.

The items were pretested to ensure that the target sentence followed sensibly from the first in all three versions. In the pretesting, 30 participants rated one of the passage versions from each item set, along with 20 nonsensible sentence pairs, using a 3-point scale ( $1=$ The sentence pair makes no sense; $2=$ I'm not sure if the pair makes sense; 3 = The sentence pair makes sense). Each of the experimental items contained an appropriately placed connective (e.g., an adversative connective was placed between adversatively related sentences). An item was declared as acceptable if at least $80 \%$ of the raters gave the highest rating (3). Seventy-three of the $90 \mathrm{ex}$ perimental sentences $(81 \%)$ met this criterion. The remaining 17 items were then repeatedly modified and evaluated by a single judge in the same manner until they were acceptable.

Table 3

Sample Items Used in Experiments 2 and 3

\begin{tabular}{|c|c|}
\hline Target Type & Item \\
\hline Additive & $\begin{array}{l}\text { Manny informed his staff about the garage sale. } \\
\text { (Consequently, However, no connective) }\end{array}$ \\
\hline Causal & $\begin{array}{l}\text { Manny needed to publicize the garage sale. } \\
\text { (Moreover, However, no connective) }\end{array}$ \\
\hline Adversative & $\begin{array}{l}\text { Manny forgot to publicize the garage sale in the paper. } \\
\text { (Moreover, Consequently, no connective) }\end{array}$ \\
\hline Target & (H)he arranged for flyers to be made. \\
\hline Additive & $\begin{array}{l}\text { John responded to the department store's unethical hiring } \\
\text { practices by writing his congressman. } \\
\text { (So, Nevertheless, no connective) }\end{array}$ \\
\hline Causal & $\begin{array}{l}\text { John found out that the department store in the mall re- } \\
\text { fused to hire minorities. } \\
\text { (Furthermore, Nevertheless, no connective) }\end{array}$ \\
\hline Adversative & $\begin{array}{l}\text { John found it difficult to go against the department store's } \\
\text { unethical hiring practices. } \\
\text { (Furthermore, So, no connective) }\end{array}$ \\
\hline Target & (H)he decided to boycott the store. \\
\hline Additive & $\begin{array}{l}\text { Ronny cleaned up the house for his girlfriend's visit. } \\
\text { (So, Nevertheless, no connective) }\end{array}$ \\
\hline Causal & $\begin{array}{l}\text { Ronny wanted to arrange a surprise for his girlfriend's visit. } \\
\text { (Also, Nevertheless, no connective) }\end{array}$ \\
\hline Adversative & $\begin{array}{l}\text { Ronny had little time to arrange a surprise for his girl- } \\
\text { friend's visit. } \\
\text { (Also, So, no connective) }\end{array}$ \\
\hline ange & $(\mathrm{H})$ he bought her a beautiful bouquet of flowers. \\
\hline
\end{tabular}


The same logical connectives that were used in Experiment 1 were used in Experiments 2 and 3 . The number of items in which each connective was used (out of 30 , in parentheses) was as follows: 1. Additive connectives - moreover (7); furthermore (8); also (6); and and (9). 2. Causal connectives-therefore (7); so (8); thus (7); and consequently (7). 3. Adversative connectives-yet (6); nevertheless (8); however (8); and but (7).

In addition to the experimental items, 63 filler sentence pairs were created. These pairs were sensible and covered many different narrative topics. Approximately one fourth of these items contained a verbal connector that preceded the second sentence. In addition, half of the filler pairs were followed by a yes/no comprehension question.

Design. Each subject was randomly assigned to one of three groups $(n=15)$. The additive group read the additive version of each of the 30 experimental items. The causal and the adversative groups read the causal and adversative versions, respectively. For each group, the experimental items rotated through three withinsubjects conditions: a no-connective condition, in which the target sentence was not preceded by a connective; and two inappropriate connective conditions, in which the connective preceding the target sentence indicated a different relation than that conveyed by the semantics of the sentence pair (for example, But appearing between two additively related sentences). For the additive group, the two inappropriate connective conditions were causals and adversatives; for the causal group, they were additives and adversatives; and for the adversative group, they were additives and causals. The connective instances used in each category were equally distributed across the items. For each group, the 30 items were counterbalanced across the three conditions, yielding a design with 10 items per condition.

Procedure. After being seated in front of a computer, participants were told that they would be reading a series of short passages, and that a comprehension question would follow a subset of those passages. They would not know in advance which passages would be followed by a question. Participants were told to read each item carefully in order to respond correctly to the question should they receive one. Each trial began with a "ready" signal. To start a trial, the participant pushed the Enter key to clear the screen and bring on the first line of a passage. Each line of text appeared in the middle of the CRT screen. After reading each line at their own pace, participants pushed either the slash $(/)$ or $z$ key to replace the current line of the passage with the next line. The lines from each passage were approximately equal in length. In passages containing connectives, the first word of the target sentence (the connective) appeared at the end of the immediately preceding line so that the target line contained identical text across conditions. The reading time for each line was computed as the time between keypresses.

On filler trials followed by a question, the last line of the passage was followed by a row of question marks (???) that appeared for $500 \mathrm{msec}$. Immediately thereafter, a comprehension question appeared on the screen. Participants answered by pressing a key marked "yes" (/) or "no" (z). The experimental session began with 3 practice passages followed by 90 passages. The reading phase of the experiment lasted approximately $20 \mathrm{~min}$.

Immediately after completing the reading task, participants were presented with an unexpected memory test. They were given a booklet containing the first sentence of each of the 30 experimental items followed by a blank line. Participants were instructed to write down what they could remember of the target sentence using the first sentence as a cue. They were given unlimited time to do this task. After completing the recall test, they were debriefed and dismissed.

\section{Results and Discussion}

Analyses of reading times. Mean RTs on the target line in each condition were computed after outliers were removed ( $3.2 \%$ of the data). Outliers were the RTs exceeding the 25 th or 75 th percentiles by the product of 1.5 and the interquartile range for each participant in each condition (Tukey, 1977). Table 4 displays the means. For each group, the inappropriate connective conditions led to longer RTs than their respective control condition (no connective).

Three ANOVAs were conducted on these data, each for the purpose of examining whether or not each type of connective caused interference compared with the noconnective condition. As before, a significance level of .05 was adopted. The first ANOVA focused on the data from the causal and adversative target groups in order to examine the impact of the inappropriate additive connectives. Across both groups, target sentence RTs following an inappropriate additive connective $(M=2,265 \mathrm{msec})$ were significantly longer than those following a no connective $\left[M=2,080 \mathrm{msec} ; F_{1}(1,28)=5.9, M S_{\mathrm{e}}=\right.$ $\left.86,609.6 ; F_{2}(1,58)=5.05, M S_{\mathrm{e}}=219,729\right] .\left(F_{1}\right.$ denotes analyses based on participants; $F_{2}$ denotes analyses based on items.) This pattern emerged for both groups, as indicated by a nonsignificant interaction between target sentence group and connective condition (both $F \mathrm{~s}<1$ ). The second ANOVA examined the impact of the inappropriate causal connectives by focusing on the data from the additive and adversative target groups. RTs on target sentences following inappropriate causals ( $M=2,238 \mathrm{msec}$ ) were marginally significantly longer than those following no connective $\left[M=2,067 \mathrm{msec} ; F_{1}(1,28)=3.52, M S_{\mathrm{e}}=\right.$ $124,633.7, p=.07 ; F_{2}(1,58)=3.55, M S_{\mathrm{e}}=302,023$, $p=.065]$. Similarly, this pattern emerged for both participant groups (interaction $F \mathbf{s}<1$ ). The data from the additive and causal target groups indicated that RTs on target sentences following erroneous adversative connectives were longer than those following no connective $\left[2,347\right.$ vs. $1,932 \mathrm{msec}$, respectively; $F_{1}(1,28)=15.68$, $\left.M S_{\mathrm{e}}=164,515 ; F_{2}(1,58)=60.26, M S_{\mathrm{e}}=89,478\right]$. The group $\times$ connective condition interaction was nonsignificant in the participants analysis $\left[F_{1}(1,28)=1.06\right.$,

Table 4

Mean Reading Times and Standard Deviations (in Milliseconds) of Target Line by Subject Group: Experiment 2

\begin{tabular}{|c|c|c|c|c|c|c|c|c|}
\hline \multirow[b]{3}{*}{ Target } & \multicolumn{8}{|c|}{ Erroneous Connective } \\
\hline & \multicolumn{2}{|c|}{ No Connective } & \multicolumn{2}{|c|}{ Causal } & \multicolumn{2}{|c|}{ Additive } & \multicolumn{2}{|c|}{ Adversative } \\
\hline & $M$ & $S D$ & $M$ & $\overline{S D}$ & $\bar{M}$ & $\overline{S D}$ & $M$ & $S D$ \\
\hline Additive & 1,918 & 250 & 2,029 & 379 & - & - & 2,225 & 512 \\
\hline Causal & 1,946 & 483 & - & $\ldots$ & 2,128 & 524 & 2,468 & 875 \\
\hline Adversative & 2,215 & 757 & 2,446 & 918 & 2,402 & 839 & - & - \\
\hline
\end{tabular}


$\left.M S_{\mathrm{e}}=164,515, p=.3\right]$, but significant in the items analysis $\left[F_{2}(1,58)=4.26, M S_{\mathrm{e}}=89,478\right]$.

The analyses above confirmed predictions that additive, causal, and adversative connectives all led to reading difficulty on the sentence following the connective when that sentence conveyed a relation to the previous sentence that did not match that dictated by the connective. Furthermore, the lack of an interaction in each analysis suggests that the interference associated with each type of connective occurred across at least two types of intersentential relations. These results are consistent with the findings of Experiment 1 and other research that has showed that readers are knowledgeable of the meanings of connectives (e.g., Goldman \& Murray, 1992). Indeed, processing disruption obtained even when both the connective and the subsequent text pertained to continuous relations (i.e., when the connective was additive or causal and the subsequent text reflected a causal or additive relation, respectively). This finding indicates that readers are sensitive to the nuances of meaning conveyed by specific connectives, and that these nuances are activated on line. Together, the analyses reported above support the notion that after encoding a connective, readers generate an expectation that the subsequent text will convey a particular relation to the previous text. Reading difficulty is the result of that expectation not being met.

To test the prediction that inappropriately placed adversative connectives will cause greater processing disruption than additives or causals, the difference between RTs for the two inappropriate connective conditions were compared. If adversative connectives influence the processing of subsequent text material to a greater extent than additives or causals, RTs in the inappropriate adversative condition should be longer than those in inappropriate additive or causal conditions.

This prediction was confirmed. In the additive group, the RT for the adversative condition $(2,225 \mathrm{msec})$ was significantly longer than that for the causal connective condition $\left[2,025 \mathrm{msec} ; t_{1}(14)=2.33, S E_{\mathrm{dm}}=84.02 ; t_{2}(29)=\right.$ $\left.2.39, S E_{\mathrm{dm}}=75.75\right]$. Similarly, in the causal group, the RT associated with adversative connectives $(2,468 \mathrm{msec})$ was longer than that of additive connectives $[2,128 \mathrm{msec}$; $t_{1}(14)=2.06, p<.06, S E_{\mathrm{dm}}=164.99 ; t_{2}(29)=3.72$, $\left.S E_{\mathrm{dm}}=92.47\right]$. The data from the adversative group showed a nonsignificant difference between the RTs associated with the additive connectives $(2,402 \mathrm{msec})$ and the causal connectives $(2,446 \mathrm{msec}$; both $t \mathrm{~s}<1)$. In summary, in line with the continuity hypothesis, inappropri- ately placed adversative connectives caused more RT disruption than either inappropriately placed additive or causal connectives.

Analysis of cued-recall data. To examine the memory effects of the erroneous connectives, all items were scored for gist recall. Each item was scored as a 1 if the gist of the target line was recalled and a 0 if the gist was not recalled. An independent rater and the experimenter each scored one third of the protocols, and agreement was $95 \%$. The remaining protocols were scored by the experimenter. The mean number of recalled items in each condition (maximum $=10$ ) for each participant group is displayed in Table 5; the means appear to be quite similar. However, the same ANOVAs as those executed with the RT data were conducted on the memory data. As the means indicate, no significant findings emerged in the analyses. Even though the inappropriately placed connectives significantly affected RT, it appears that subsequent recall was not affected by the incongruency between the connective and the intersentential context.

\section{EXPERIMENT 3}

The purpose of Experiment 3 was to test the extent to which the findings of Experiment 2 extend to readers' conscious perceptions of sentence coherence. Participants rated the same experimental items used in Experiment 2 in terms of the degree to which the second sentence sensibly followed the preceding sentence. It was hypothesized that the extent to which additive, causal, and adversative connectives indicate continuity or discontinuity should be reflected in participants' ratings of the sensibility of the postconnective sentence. The findings of Experiment 2 suggested that participants should perceive a sentence that follows an inappropriate connective as following less sensibly from the preceding sentence than a target preceded by no connective. However, as predicted in Experiment 2, this effect should be more pronounced for adversatives than for additives and causals.

\section{Method}

Participants. Forty-five undergraduates enrolled in introductory psychology participated in this study in exchange for extra credit. Participants were randomly assigned to one of three groups $(n=15)$.

Design, Materials, and Procedure. The design and experimental materials used in Experiment 3 were identical to those used in Experiment 2. Participants were presented with the 30 experimental sentence pairs along with the 20 anomalous (filler) sentence pairs used in the item pretesting. The 50 sentence pairs were placed in a

Table 5

Mean Proportion and Standard Deviations of Items Recalled by Condition and Participant Group (Maximum $=10$ ): Experiment 2

\begin{tabular}{|c|c|c|c|c|c|c|c|c|}
\hline \multirow[b]{3}{*}{ Group } & \multicolumn{8}{|c|}{ Erroneous Connective } \\
\hline & \multicolumn{2}{|c|}{ No Connective } & \multicolumn{2}{|c|}{ Causal } & \multicolumn{2}{|c|}{ Additive } & \multicolumn{2}{|c|}{ Adversativ } \\
\hline & $M$ & $S D$ & $M$ & $S D$ & $M$ & $S D$ & $M$ & $S D$ \\
\hline Additive target & .47 & .19 & .53 & .11 & - & - & .47 & .21 \\
\hline Causal target & .55 & .22 & - & - & .51 & .21 & .49 & .21 \\
\hline Adversative target & .59 & .19 & .57 & .22 & .50 & .20 & 一 & - \\
\hline
\end{tabular}


booklet in random order. A blank line was placed next to each sentence pair for participants to write a number from 1 to 5 reflecting the extent to which the second sentence of the pair "followed sensibly" from the first sentence $(1=$ doesn't follow at all; $5=$ follows extremely well). Participants were given unlimited time for the task.

\section{Results and Discussion}

The mean ratings for each of the two inappropriate connective conditions along with the no-connective control were computed for each participant. The means for each group are displayed in Table 6 . Higher values in the table reflect greater perceived sensibility of the target sentence. With one exception, the means of both inappropriate connective conditions for each subject group yielded lower ratings than those associated with the no-connective condition.

To investigate the reliability of this pattern, the analyses executed on the RT data in Experiment 2 were repeated here. The data from the causal and adversative target groups indicated that inappropriate additive connectives caused a marginally significant decrease in sensibility than did the no-connective control $\left[F_{1}(1,28)=3.27\right.$, $M S_{\mathrm{e}}=.094, p=.08 ; F_{2}(1,58)=2.24, M S_{\mathrm{e}}=.275, p=$ .14]. This pattern differed slightly between the groups, as indicated by the marginally significant interaction in the participants analysis $\left[F_{\mathrm{l}}(1,28)=3.58, M S_{\mathrm{e}}=.094, p=\right.$ $\left..07 ; F_{2}(1,58)=2.45, M S_{\mathrm{e}}=.275, p=.12\right]$. The data from the additive and adversative target groups showed, in contrast to the findings from Experiment 2, that inappropriately placed causal connectives did not lead to a significantly lower rating of the target sentence than the no-connective control $\left(F_{1}\right.$ and $\left.F_{2}<1\right)$. This pattern was similar for both groups (interaction $F \mathrm{~s}<1$ ). And, as expected, the ratings from the additive and causal groups showed that the presence of inappropriate adversative connectives led to lower sensibility ratings compared with the control $\left[F_{1}(1,28)=34.10, M S_{\mathrm{e}}=.245 ; F_{2}(1,58)=\right.$ $\left.69.32, M S_{\mathrm{e}}=.241\right]$. The group $\times$ connective condition interaction was significant only in the items analysis $\left[F_{1}(1,28)=2.45, M S_{\mathrm{e}}=2.45, p=.13 ; F_{2}(1,58)=4.97\right.$, $\left.M S_{\mathrm{e}}=.241\right]$.

To test the prediction that inappropriate adversative connectives would affect sensibility ratings to a greater extent than inappropriate additives or causals, the same condition comparisons executed within each group were executed with the ratings. The data from the additive group showed that targets following erroneously placed adversatives $(M=2.99)$ were rated significantly lower than tar- gets following erroneous causal connectives $[M=3.41$; $\left.t_{1}(14)=2.43, S E_{\mathrm{dm}}=.17 ; t_{2}(29)=3.31, S E_{\mathrm{dm}}=.13\right]$. Similarly, ratings from the causal group indicated that targets following adversative connectives $(M=3.37)$ were rated lower than those following additives $[M=4.03$; $\left.t_{1}(14)=4.14, S E_{\mathrm{dm}}=.16 ; t_{2}(29)=5.39, S E_{\mathrm{dm}}=.12\right]$. In the adversative group, no difference was found between the ratings of the additives $(M=2.53)$ and the causals $(M=2.48$; both $t \mathrm{~s}<1)$.

In general, the presence of incorrect connectives led to lower ratings of passage coherence, but this effect was weaker with connectives that signaled continuity (additives and causals) than with adversatives. Consistent with the findings from Experiment 2, inappropriately placed additives led to lower ratings of coherence than did the no-connective control. However, the effect disappeared when the target sentence was preceded by an incorrect causal connective. In contrast to the RT data from Experiment 2 , readers did not find inappropriately placed causal connectives to be more disruptive than no connective at all. However, the critical prediction in line with the continuity hypothesis was confirmed: that erroneously placed adversative connectives would lead to greater disruption in perceived coherence than would erroneously placed additive and causal connectives.

\section{GENERAL DISCUSSION}

The present research adopts the view that readers have a default assumption to interpret subsequent sentences in narrative text in a continuous manner (Segal et al., 1991). Connectives are text devices that signal the relation between the immediately preceding sentence or clause with the next sentence or clause. Research in the areas of text processing and language development has indicated that the interpretation and use of connectives during reading is a critical component of text processing. Whereas previous research on connective processing has indicated that different types of connectives benefit integrative processing to various degrees, no common mechanism has been hypothesized that explains which connectives will impact processing the most. The purpose of the present research was to propose such a mechanism.

It was hypothesized that a connective would impact processing to the extent that it signals discontinuity in the text. If readers assume that successive sentences will continue in a linear, forward manner, then any text device that signals a disruption in this continuity is likely,

Table 6

Mean Ratings and Standard Deviations of Target Size by Condition and Participant Group: Experiment 3

\begin{tabular}{|c|c|c|c|c|c|c|c|c|c|c|}
\hline \multirow[b]{3}{*}{ Group } & \multicolumn{10}{|c|}{ Erroneous Connective } \\
\hline & \multicolumn{2}{|c|}{ No Connective } & \multicolumn{2}{|c|}{ Causal } & \multicolumn{2}{|c|}{ Additive } & \multicolumn{2}{|c|}{ Adversative } & \multicolumn{2}{|c|}{ Filler } \\
\hline & $M$ & $S D$ & $M$ & $S D$ & $M$ & $S D$ & $M$ & $S D$ & $M$ & $S D$ \\
\hline Additive target & 3.53 & .61 & 3.41 & .54 & - & - & 2.99 & .61 & 2.01 & .32 \\
\hline Causal target & 4.32 & .35 & - & - & 4.03 & .51 & 3.37 & .87 & 2.12 & .27 \\
\hline Adversative target & 2.53 & .39 & 2.48 & .47 & 2.53 & .60 & - & - & 2.01 & .30 \\
\hline
\end{tabular}


in theory, to impact processing more than would a device that signals continuity. Whereas this hypothesis was specifically tested in Experiments 2 and 3, the results of Experiment 1 showed that readers use logical connectives as indicators of continuity or discontinuity. When an additive or causal connective was provided as the first word of a sentence, participants generated sentences that depicted a continuous progression of the action depicted in the stimulus sentence. When an adversative connective was provided, participants generated sentences that reflected discontinuous relations.

The extent to which different types of connectives impact on-line processing was examined in Experiments 2 and 3. These experiments provided a follow-up to research showing that when used appropriately, adversative connectives have a greater impact on on-line processing than do additive or causal connectives (Murray, 1994). It was reasoned that the impact of adversative connectives was based on the fact that these connectives are clear indicators of discontinuity in the discourse. Because discontinuity is the exception rather than the rule, any signal of discontinuity is likely to impact on-line processing to a greater extent than would a signal of continuity.

This idea was tested in the present experiments by assessing the impact of inappropriately placed additive, causal, and adversative connectives. It was hypothesized that just as adversatives were associated with the greatest degree of processing facilitation when they were used appropriately, so they should lead to the greatest processing disruption when used inappropriately. This prediction was confirmed when disruption was measured on line (using RT) in Experiment 2 and off-line (using coherence ratings) in Experiment 3. Furthermore, no difference in processing disruption was observed between additive and causal connectives in either experiment. Even though readers are aware of the unique types of relations signaled by these connectives, the fact that these two types of connectives were not differentially disruptive suggests that the relation readers exploit for the purposes of on-line processing is that of continuity rather than a more specific relation (e.g., general elaboration in the case of additives or cause-effect in the case of causals).

As mentioned in the introduction to this article, other researchers have presented findings that might appear to be at odds with the continuity hypothesis (Caron et al., 1988; Haberlandt, 1982; Millis \& Just, 1994). In some cases, however, the text settings used in these studies did not conform to the setting to which the continuity hypothesis applies (i.e., adjacent sentences that convey a coherent relation). In other cases, such as the benefits observed with the connective because, the continuity hypothesis does account for the observed facilitation (see Introduction).

The present findings indicate that the continuity hypothesis may influence on-line interclausal integration. However, a precise understanding of how connectives influence integrative processing is not yet certain. In their connective integration model, Millis and Just (1994) have argued that connectives signal to the reader that the upcom- ing clause is to be integrated with the immediately preceding clause. The model states that when a reader encodes a connective, the representation of Clause 1 is set aside until reaching the final word of Clause 2. At that point, Clause 1 is reactivated and is integrated with Clause 2 into a unified representation. In the absence of a connective, integration takes place while Clause 2 is being read. This reactivation hypothesis is not inconsistent with the present findings, which indicate that connectives influence the processing of the line that follows them. However, the model does not explain how the different meaning constraints associated with different connectives (e.g., continuity/discontinuity) may differentially impact interclause integration. In order to account for the present findings as well as other recent data (e.g., Murray, 1994), the model needs to explain how connectives that signal continuity do not necessarily lead to a shorter overall RT on Clause 2.

Without a doubt, more testing of the continuity hyputhesis is needed for it to be considered as an accurate depiction of connective influence. In addition to testing how the hypothesis fares in different text settings (e.g., expository settings, different text lengths), future research needs to investigate the effects of specific connectives rather than specific types of connectives. Clearly, as in the case of because, all connectives within a particular classification do not signal continuity/discontinuity to the same extent. Furthermore, Millis and Just (1994) have argued that the connective-initiated integrative processes occur at the end of Clause 2, during sentence wrap-up. Whether this pattern obtains in the context of connectives that signal continuity remains an empirical question. In addition, the precise nature of the integrative processes that are presumed to occur at sentence wrap-up are still unclear. The plausibility of this and other possible mechanisms should be explored in future research.

\section{REFERENCES}

Bloom, L., Lahey, M., Hood, L., Lifter, K., \& Fiess, K. (1980). Complex sentences: Acquisition of syntactic connectives and the semantic relations they encode. Journal of Child Language, 7, 235-261.

Caron, J., Micko, H. C., \& Thuring, M. (1988). Conjunctions and the recall of composite sentences. Journal of Memory \& Language, 27, 309-323.

Francis, W. N., \& KUČERA, H. (1982). Frequency analysis of English usage. Boston: Houghton Mifflin.

Golding, J. M., Millis, K. M., Hauselt, J., \& Sego, S. A. (1994). The effect of connectives and causal relatedness on text comprehension In R. F. Lorch, Jr., \& E. J. O'Brien (Eds.), Sources of coherence in reading (pp. 127-143). Hillsdale, $\mathrm{NJ}$ : Erlbaum.

Goldman, S. R., \& MURRaY, J. D. (1992). Knowledge of connectors as cohesion devices in text: A comparative study of native English and ESL speakers. Journal of Educational Psychology, 84, 504-519.

HABERLANDT, K. (1982). Reader expectations in text comprehension. In J. Le Ny \& W. Kintsch (Eds.), Language and comprehension. Amsterdam: North-Holland.

Halliday, M. A. K., \& Hasan, R. (1976). Cohesion in English. London: Longman.

JOHNSON-LAIRD, P. N. (1983). Mental models: Towards a cognitive science of language, inference, and consciousness. Cambridge, MA: Harvard University Press.

KATZ, E. W., \& BRENT, S. B. (1968). Understanding connectives. Journal of Verbal Learning \& Verbal Behavior, 11, 501-509. 
KinTsCh, W., \& van DiJK, T. A. (1978). Toward a model of text comprehension and production. Psychological Review, 85, 363-394.

LinTERMANN-RyGH, I. (1985). Connector density: An indicator of essay quality. Text, $5,347-357$.

MCCluRE, E., \& Geva, E. (1983). The development of the cohesive use of adversative conjunctions in discourse. Discourse Processes, 6 411-432.

Millis, K. K., Graesser, A. C., \& Haberlandt, K. (1993). The impact of connectives for memory for expository texts. Applied Cognitive Psychology, 7, 317-339.

Millis, K. K., \& Just, M. A. (1994). The influence of connectives on sentence comprehension. Journal of Memory \& Language, 33, 128-147.

MurRay, J. D. (1994). Logical connectives and local coherence. In R. F. Lorch \& E. J. O'Brien (Eds.), Sources of cohesion in text comprehension (pp. 107-125). Hillsdale, NJ: Erlbaum.

Peterson, C., \& McCabe, A. (1987). The connective and: Do older children use it less as they learn other connectives? Journal of Child Language, 14, 375-381.
Segal, E. M., Duchan, J. F., \& Scott, P. J. (1991). The role of interclausal connectives in narrative structuring: Evidence from adults' interpretations of simple stories, Discourse Processes, 14, 27-54.

TUKEY, J. W. (1977). Exploratory data analysis. Reading, MA: AddisonWesley.

\section{NOTE}

1. Even though the absolute level of recall was identical for the connective present and absent groups, adversative connectives were assumed to facilitate memory because the target line RTs were shorter for the connectives present than for the connectives absent group. For additive and causal targets, the relationship between processing effort and recall did not differ for the different groups.

(Manuscript received October 3, 1995 revision accepted for publication February 19, 1996.) 